\title{
A case of metachronous aneurysm of the infrarenal abdominal aorta
}

\author{
Fraser C. Millard and Simon J.A. Powis \\ Department of Surgery, Northampton General Hospital, Cliftonville, Northampton NN1 5BD, UK.
}

\begin{abstract}
Summary: The case history of an 84 year old woman presenting with a metachronous aneurysm of the infrarenal abdominal aorta is presented. At operation this second aneurysm was replaced by a further straight tube Dacron inlay graft.

This report raises the important question as to whether the whole of the infrarenal aorta should be replaced when resecting an infrarenal abdominal aortic aneurysm, rather than the more conventional approach of suturing the graft to the neck of the aneurysm above and the aortic bifurcation below.
\end{abstract}

\section{Introduction}

Arterial aneurysms are occasionally multifocal, particularly in patients with aortic aneurysms located in the thorax and in those with aneurysms caused by Marfan's syndrome, dissection, and infection, and those occurring after previous operation. $^{1}$ However, while late deaths following successful resection of abdominal aortic aneurysms have been reported from rupture of a second dissecting or thoracic aneurysm, ${ }^{2}$ metachronous infrarenal aneurysm following previous replacement of an infrarenal aortic aneurysm with a Dacron inlay graft has not been described.

Such a case is presented and its implications discussed.

\section{Case report}

At routine review following aortic aneurysmectomy 7 years previously, an 84 year old woman gave a history of increasing abdominal swelling. At her first operation, a leaking infrarenal aortic aneurysm had been replaced with a straight tube Dacron inlay graft, the aneurysm neck being some $4-5 \mathrm{~cm}$ below the renal arteries. On examination, she had a tender epigastric mass which exhibited expansile pulsation. Ultrasound scan showed this to be an aneurysm of $5.8 \mathrm{~cm}$ diameter, while digital subtraction arteriogram confirmed this metachronous aneurysm to be

Correspondence: F.C. Millard, F.R.C.S., X-Ray Department, Royal Hallamshire Hospital, Glossop Road, Sheffield S10 2JF, UK.

Accepted: 22 December 1987 arising from the infrarenal aorta above her original prosthesis.

In view of her good general health, and the tenderness associated with the aneurysm, it was decided to proceed to elective operation when the aneurysm was found to have a neck immediately below the renal arteries (Figure 1). This was a true aneurysm involving the infrarenal aortic remnant from her previous surgery, the suture line being quite uninvolved. This metachronous aneurysm was

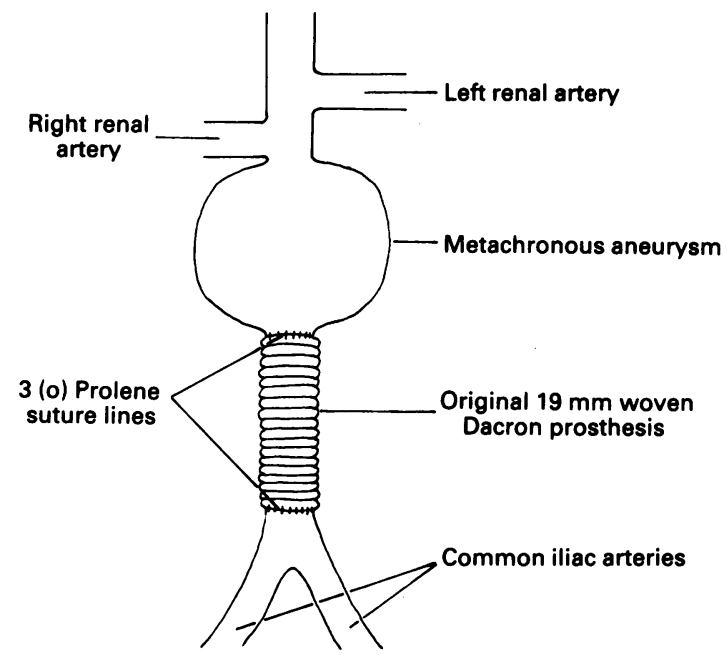

Figure 1 Diagram of metachronous aneurysm of infrarenal abdominal aorta. For clarity a graft repair by end to end anastomosis rather than by inlay technique is shown.

(C) The Fellowship of Postgraduate Medicine, 1988 
replaced by a further straight tube Dacron inlay graft from its neck to the origin of the original prosthesis without any technical difficulties. However her immediate postoperative recovery was complicated by a consumption coagulopathy from which she died.

\section{Discussion}

The development and improvement of prosthetic grafts, operative techniques, and perioperative care, have made elective abdominal aortic aneurysm repair a safe operation. Earlier reports from large centres showed a mortality rate of about $10 \%$ for elective repair of infrarenal abdominal aortic aneurysms, ${ }^{3.4}$ while figures of less than $5 \%$ are now being published. ${ }^{5}$ By contrast, the average mortality rate for ruptured infrarenal abdominal aortic aneurysms is about $50 \%$, and these survivors are only a small minority of the total number of

\section{References}

1. Crawford, E.S. \& Cohen, E.S. Aortic aneurysms: a multifocal disease. Arch Surg 1982, 117: 1393-1400.

2. MacVaugh, H. \& Roberts, B. Results of resection of abdominal aortic aneurysms. Surg Gynecol Obstet 1961, 113: 17-23.

3. Gordon-Smith, I.C., Taylor, E.W., Nicolaides, A.N., Golcman, L., Kenyon, J.R. \& Eastcott, H.H.G. Management of abdominal aortic aneurysm. Br J Surg 1978, 65: 834-838.

4. De Bakey, M.E., Crawford, E.S., Cooley, D.A., Morris, G.C., Royster, T.S. \& Abbott, W.P. Aneurysm of the abdominal aorta. Ann Surg 1964, 160: 622-639. people in the community whose aneurysms rupture. ${ }^{6}$ Therefore the elective repair of infrarenal abdominal aortic aneurysms is becoming an increasingly common operation.

The late complication reported here has not been described previously, and therefore its true incidence is not known. It is, however, likely to become increasingly well recognized. Conventionally, when resecting an infrarenal aortic aneurysm, the aneurysm alone is excised, the proximal anastomosis being sutured at the neck of the aneurysm. ${ }^{7,8}$ However, as this case illustrates, when the neck of the aneurysm lies well below the renal arteries, further aneurysm formation may then occur in the infrarenal aortic remnant. Therefore this raises the important question as to whether the whole of the infrarenal aorta should be replaced in such cases, rather than the more conventional technique of suturing the graft to the neck of the aneurysm sac above and the bifurcation below.

5. Hollier, L.H. Surgical management of abdominal aortic aneurysm in the high risk patient. Surg Clin North Am 1986, 66: 269-279.

6. Armour, R.H. Survivors of ruptured abdominal aortic aneurysm: the iceberg's tip. Br Med J 1977 2: 1055 1057.

7. DeWeese, J.A. The treatment of abdominal aortic aneurysms. In: Rob, C. \& Smith, Sir R. (eds) Atlas of General Surgery compiled by Dudley, H., Carter, D.C., Russell, R.C.G. Butterworths, London and Boston, 1986, pp. 862-874.

8. Wyatt, A.P. Presentation and management of aneurysms. Ann R Coll Surg Engl 1976, 58: 52-61. 